\title{
Allostery in membrane proteins
}

\author{
Zoe Cournia $^{1^{*}}$ and Alexios Chatzigoulas ${ }^{1,2}$ \\ ${ }^{1}$ Biomedical Research Foundation, Academy of Athens, 11527 Athens, Greece \\ ${ }^{2}$ Department of Informatics and Telecommunications, National and Kapodistrian University of Athens, \\ 16784 Athens, Greece
}

\begin{abstract}
Membrane proteins are an integral part of signal transduction. To signal, membrane proteins must interact with a variety of lipid species, effectors, and other proteins in the biological membrane leading to an immense number of possible interactions. Despite this inherent complexity, accurate control of signaling must take place. By allowing proteins to adopt a multiplicity of conformations in a process known as allostery, nature is able to transmit a signal from one protein site to another distal, functional site, allowing for modulation of protein properties and regulation of activity. In recent years, an increasing number of reports have pointed to common mechanisms governing the allosteric modulation of membrane proteins, including conformational selection, oligomerization, and the modulation of allosteric sites. In this report, we summarize recent advances in membrane protein allostery.
\end{abstract}

\section{Introduction}

Signal transduction is largely controlled by proteins embedded in or associated with membranes. Although membrane proteins play an important role in cellular signaling, they have only recently been recognized to act as allosteric bodies. In fact three out of the four major receptor families that act allosterically are membrane proteins, i.e. ligand- and voltage-gated ion channels, G-protein-coupled receptors (GPCRs), and receptor tyrosine kinases. [1] Membrane protein regulation can be facilitated by signaling molecules integral to the cell membrane and other effectors. Over the past decade, a wealth of protein structural data has become readily available including many membrane protein classes, e.g., GPCRs, ion channels and peripheral membrane proteins, which has enabled the elucidation of allosteric characteristics of many membrane proteins. With allostery gaining increasing attention as an opportunity to modulate protein function, and with membrane proteins being a significant fraction of the pharmaceutical targets, exciting opportunities for drug discovery have opened up.

Allostery can be defined as the functional change of the orthosteric site generated by a perturbation produced from the binding of an effector to a distant site (allosteric site). The effect at the allosteric site is linked to the active site by small 
conformational changes and dynamics that transmit the allosteric effect in a wavelike manner along pathways of amino acids (allosteric pathways) in the protein, thus regulating its activity.[2] This biological phenomenon was first introduced by Cristian Bohr over a century ago, who observed positive cooperativity of oxygen binding to the hemoglobin protein.[3] Since then, numerous approaches have been emerged to explain this phenomenon [4-7] until the derivation of the Monod-Wyman-Changeux (MWC) concerted model[8] introduced to describe this cooperativity, which along with the Koshland-Nemethy-Filmer (KNF) sequential model, [9] were for years the leading allosteric models.

The current approach to allostery is that a perturbation at an allosteric site in the structure leads to a shift in equilibrium populations, concluding that allostery is a property of the conformational ensemble (Figure 1A).[10-12] Based on this redistribution of the conformational ensemble, it is deduced that allostery is not only associated with the cooperativity between enzyme subunits, but it is an intrinsic property of all dynamic proteins.[1,13] Consequently, in the protein conformational ensemble different allosteric mechanisms preexist,[14] thus the binding of an allosteric modulator stabilizes the active form of the allosteric domain, which through the preexisting allosteric pathways modulates the activity (positively or negatively) of the functional domain remodeling the energy landscape.[11,15] In the absence of an agonist, the tense or inactive state dominates the conformational ensemble, while in the presence of an agonist the conformational equilibrium shifts toward the active state (Figure 1A). When agonists are present in high concentrations, a desensitized state can occur (Figure 1B). In the desensitized state, the receptor exhibits decreased response although bound to its agonist. This is an allosteric property of most membrane receptors that aids homeostasis maintenance. By excess binding of agonists, desensitization occurs by receptor uncoupling, or internalization of the membrane receptors (endocytosis), reducing their amount on the cell membrane.[16,17] 
A)

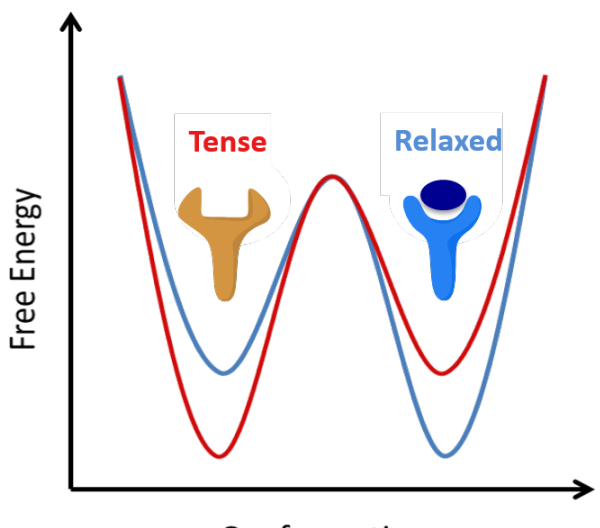

B)

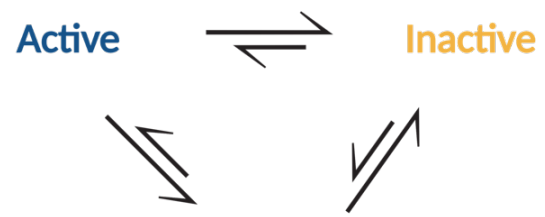

Desensitized

Figure 1. A) Allosteric proteins are found in equilibrium between an inactive (tense) state (red curve) and an active (relaxed) state. In the tense state, the protein is inactive and binds to its ligand with low affinity. In the active state the protein binds with high affinity to the ligand. A negative allosteric inhibitor can shift the population to the tense state, while a positive allosteric modulator can stabilize the relaxed state moving the conformational ensemble towards the relaxed state. This picture is consistent with the MWC model of allostery. B) In membrane proteins, excessive agonist concentrations shift the equilibrium towards a desensitized state, where the receptor activation is weakened although bound to its agonist.

In this review, we annotate the homotropic and heterotropic types of allosteric modulation and their effect on membrane proteins. We present the allosteric nature of three major transmembrane protein classes, namely GPCRs, ligand-gated ion channels, and receptor tyrosine kinases, and illustrate functional changes, which are generated allosterically. Furthermore, we report on how lipids may act as allosteric modulators on transmembrane proteins, and outline the various allosteric mechanisms of peripheral membrane proteins and lipid-anchored proteins. Finally, we highlight new drug design opportunities that may arise by allosterically modulating membrane proteins that hitherto remain undruggable.

\section{Heterotropic and homotropic membrane protein allostery}

One important observation is that allostery in membrane proteins may be exerted in two forms, heterotropic and homotropic allostery. In heterotropic allostery, binding of a negative or positive allosteric modulator (NAM or PAM, respectively) shifts the conformational ensemble to the tense or relaxed state, respectively, thereby reducing or enhancing binding of the orthosteric ligand. The tense and relaxed conformations are found in equilibrium and conformational selection is achieved by allosteric modulators. Homotropic allostery is relevant when oligomerization occurs in membranes. Binding of one ligand in one of the monomers enhances the binding of further ligands to the multiprotein complex. The sequence of events is in accordance with the MWC model of allostery (Figure 2). One example of a 
heterotropic and homotropic allosteric protein are $\alpha \beta$ T-cell receptors, where the activity of $\alpha \beta$ T-cell receptors (TCRs) is controlled by conformational switches. Binding of multivalent peptide-MHC to the TCR stabilizes the relaxed conformation, leading to TCR signaling. In contrast, cholesterol may bind to the resting conformation and stabilize the inactive state, thus acting as a negative allosteric regulator. Therefore, the MHC peptide and cholesterol function in the heterotropic allostery scheme. TCRs can also assemble into nanoclusters that allows for homotropic allostery, in which individual TCRs may positively cooperate and thus enhance the sensitivity of T cell activation.[18]

Heterotropic allostery at the membrane and selection of a conformational state
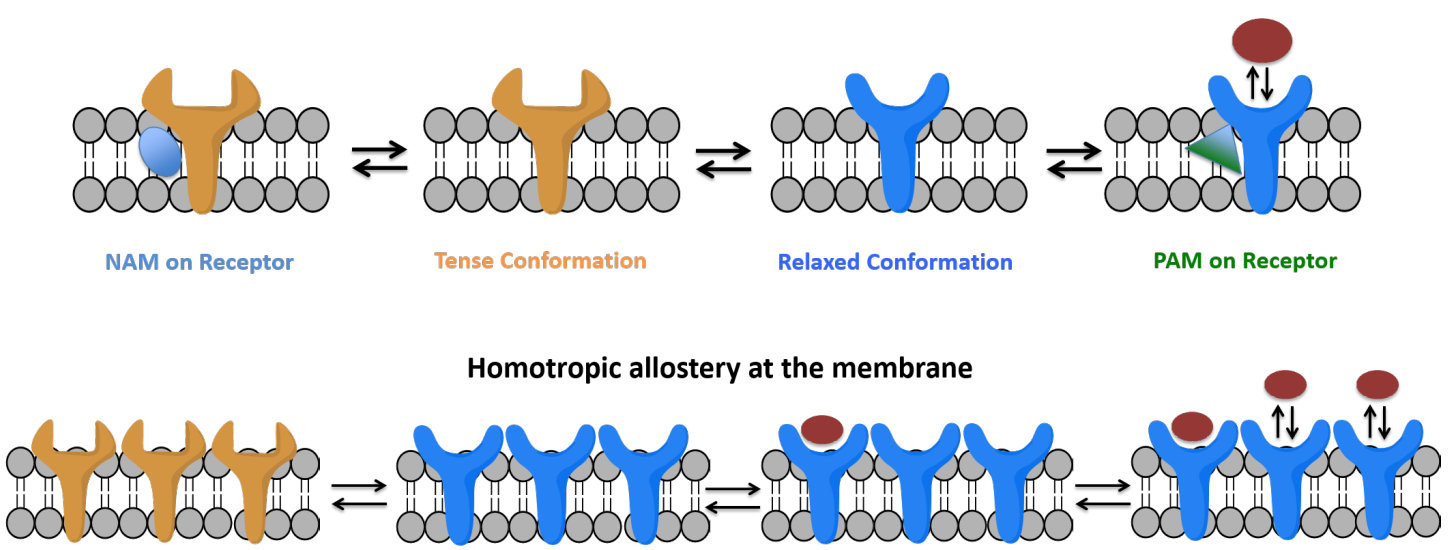

Figure 2. Schemes for heterotropic allostery (upper panel) and homotropic allostery (lower panel). Heterotropic allostery acts with negative or positive allosteric modulators, shifting the conformational ensemble towards the tense or the relaxed state, respectively. Homotropic allostery occurs when oligomerization on the membrane takes place and the cooperativity of monomers can enhance the binding of orthosteric ligands (lower panel).

\section{GPCR allosteric modulation}

One of the largest families of membrane proteins is GPCRs. The human genome encodes about 800 GPCRs with the rhodopsin-like class A receptors representing the quantitatively dominating subfamily, which include aminergic and cholinergic receptors. $[19,20]$ GPCRs are present in all cells in the body. They pass signals from a variety of external cellular messengers to modulate intracellular pathways via the activation of G-proteins and other signaling molecules. The first high-resolution 3D structural data for rhodopsin/ $\beta$-adrenergic receptors in 2007-2008 (reviewed in [21]) have enabled the elucidation of the molecular basis of GPCR allostery. GPCRs possess multiple sites capable of allosteric modulation. Three allosteric vectors, directed toward 1) the ectodomain, 2) the cytosolic face, and 3) the intramembranous faces of GPCRs, provide numerous opportunities for functional selectivity of the action of drugs.[21] Because GPCRs form ternary complexes comprising of the receptor, a ligand, and an intracellular binding partner, GPCRs may 
allosterically couple extra- and intra-cellular components serving therefore as gates to the inside of the cell. This allosteric coupling mechanism renders GPCRs dynamic entities that can be chemically controlled based on their binding partner. Because different active GPCR states exist in a conformational equilibrium [22,23], agonists may selectively activate different cellular signaling pathways depending on their chemistry, while in the case of overstimulation, G-proteins couple with arrestins, leading to GPCR desensitization.[24,25] Thus, they may stabilize a unique ensemble of active states resulting in a signal that can be biased toward a specific subset of pathways, a phenomenon termed "ligand or agonist bias".[26] Biased agonists may have superior therapeutic profiles as they can influence a specific pathway in the cell. The concept of biased agonism based on the ligand specific stabilization of a subset of receptor conformations is now well established, and first examples of biased compounds for the Angiotensin II Receptor and the $\mu$-opioid receptor entering clinical trials are promising. Dualsteric/bitopic ligands, where for example an inverse allosteric agonist and an orthosteric agonist are coupled in the same molecule, are now emerging as a new trend in GPCR modulation.[27]

The ligand-binding sites of most GPCRs are embedded within the central pocket formed by their seven transmembrane-spanning $\alpha$-helical domains. While it was believed that these binding sites are only accessible to endogenous ligands from the aqueous phase, recent work suggests that small molecules may access GPCRs by an indirect pathway through partitioning into the membrane and then reaching the binding site via lateral diffusion through the lipid bilayer.[28,29] Moreover, recent crystal structures reveal the existence of extrahelical lipid allosteric sites at the interface of the lipid-protein interface and at different bilayer depths.[28] Cholesterol and phospholipids can act as positive, negative allosteric and even orthosteric modulators of GPCRs by binding directly to receptor sites on the membrane interface or inside the receptor.[30-32] *

Finally, heterodimerization of GPCRs driven by allostery at the plasma membrane is paramount for the function of some members of this protein family. For example, it is long known that heterodimerization is crucial for the formation of a functional GABA $B$ receptor[33], but also of the A2A receptor with $D 2 R$, the A2A receptor with $\alpha$-synuclein, metabotropic glutamate receptors such as mGluR5 interaction with contactin associated protein 1, which appears crucial for the ability of mGluR5 to control memory formation in the hippocampus, mGluR2-mGluR4 heteroreceptor complexes and many others, which have been recently reported and are reviewed in Ref. [34].**

\section{Ligand- gated ion channels}

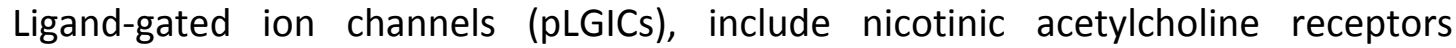
(nAChRs), serotonin (5HT3) and the inhibitory glycine, glutamate, and GABAA receptors, tetrameric ionotropic glutamate receptors, and trimeric ATP-gated P2X receptors and acid-sensing channels.[1] pLGICs play a central role in the function of 
the central nervous system by converting the binding of a neurotransmitter (e.g. acetylcholine) into an ion flux through the postsynaptic membrane. pLGICs are oligomeric assemblies that are exemplars of allosterically regulated integral membrane proteins.[35] Recent results suggest that ion gating involves a large structural reorganization of the protein mediated by two distinct quaternary transitions, a global twisting and the blooming of the extracellular domain, which can be modulated by ligand binding at the topographically distinct orthosteric and allosteric sites.[35] Latest research findings support a multisite model of transmembrane allosteric modulation, including a possible link between lipid- and receptor-based theories that could inform the development of new anesthetics.[36]* It should be noted that in high-resolution open-state GLIC structures, but not in non-conducting structures, a lipid occupies the membranefacing vestibule of the intrasubunit cavity.[37,38] In 2018, ten new X-ray structures of GLIC variants in the presence and absence of general anesthetics, including the surgical agent propofol were presented. These anesthetics can allosterically favor closed channels by binding in the pore or favor open channels via various subsites in the transmembrane domain.[39] It should be noted that agonist-bound pLGICs do not necessarily represent the physiological active state, as they may represent the agonist-bound shut-channel state (desensitized state). $[40,41]$ For the trimeric P2X channels, the key steps in the allosteric transition have been proposed to be regulated by the interfaces between different subunits.[1]

\section{Receptor Tyrosine Kinases}

58 receptor tyrosine kinases (RTKs) are encoded in membrane proteins in the human genome, with those further subdivided into 20 families. RTKs are an integral part of signaling as they function as receptors for polypeptide growth factors, cytokines, and related hormones.[1] RTKs have a multidomain ECD of variable length, which recognizes the orthosteric ligand(s), a single pass transmembrane helix, and an intracellular juxta-membrane domain (JMD) that links to the tyrosine kinase domain (TKD), which performs the catalytic function initiating signal transduction. Perhaps the most prominent member of the RTK family is the epidermal growth factor receptor (EGFR). EGFR as all protein kinases can populate two states: the active and the inactive. Recent literature [42] suggests that the ligand activates EGRF extracellularly and strongly couples across the membrane to achieve full activation. In a diseased state, where EGFR is overexpressed or mutated, EGFR can be activated independently of the ligand, mediated by intracellular kinase dimerization.

\section{Allosteric mechanisms of Peripheral Membrane Proteins and Lipid Anchored Proteins}

Allostery is not limited only to transmembrane proteins. Peripheral membrane proteins that are attached to the surface of the cell via non-bonded interactions with membrane phospholipids as well as lipid-anchored proteins are also regulated allosterically. Though not as abundant as transmembrane proteins, these two classes 
of membrane proteins contain some of the most prevalent protein drug targets: KRAS and PI3K $\alpha$, which are known oncogenes, and $\alpha$-synuclein implicated in Parkinson's disease. The lipid kinase PI3K $\alpha$ is modulated allosterically by receiving a signal from receptor tyrosine kinases and/or K-Ras binding and recruited to the plasma membrane, where it phosphorylates the PIP2 lipid to PIP3 in the presence of ATP. [43,44] A PI3K $\alpha$ hotspot mutant, H1047R, which is prevalent in breast, colon and other cancers, has been shown to act by modulating the way that the kinase interacts with the cell membrane.[45] We performed dynamical network analysis [46] of WT and H1047R mutant trajectories reported in Ref. [45] and found that $\mathrm{H} 1047$ is connected to the PI3K $\alpha$ membrane-binding loop (residues 863-873) through a pathway of ten residues, while the mutant 1047R significantly shortens this path and connects itself to the membrane-binding loop through a pathway of six residues (Figure 3).

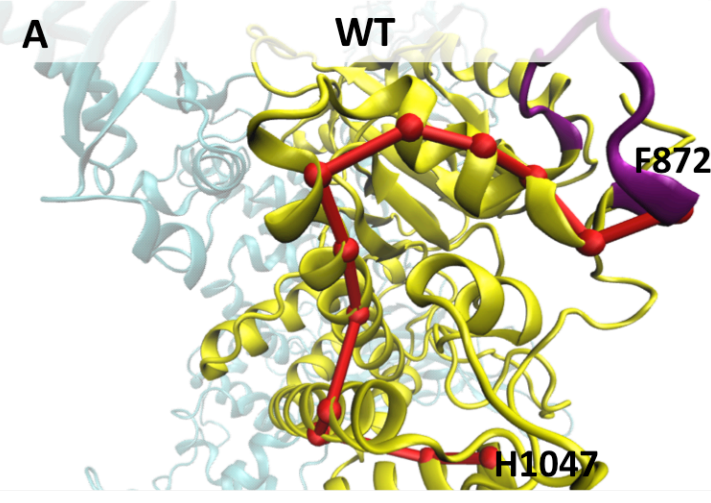

$1047 \rightarrow 1043 \rightarrow 985 \rightarrow 982 \rightarrow 979$ $\rightarrow 898 \rightarrow 894 \rightarrow 890 \rightarrow 879 \rightarrow 876$ $\rightarrow 874 \rightarrow 872$

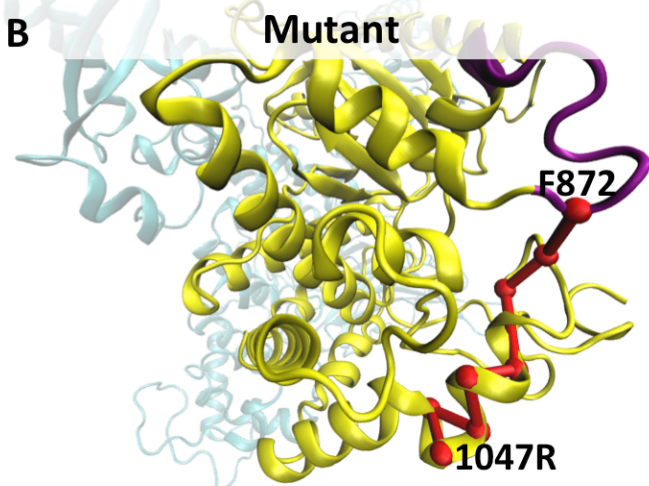

$1047 \rightarrow 1045 \rightarrow 1048 \rightarrow 1050 \rightarrow 1052$ $\rightarrow 1060 \rightarrow 1057 \rightarrow 872$

Figure 3. Dynamical network analysis was performed on PI3Ka WT and H1047R mutant trajectories reported in Ref. [45]. H1047, which belongs to the C-terminal tail of PI3Ka, is connected to the PI3Ka membrane-binding loop (residues 863-873) through a pathway of ten residues. The mutant 1047R significantly shortens this path and connects itself to the membrane-binding loop through a pathway of six residues. The kinase domain is colored in yellow, the membrane-binding loop in purple and the pathway identified by dynamical network analysis in red.

The four RAS isoforms KRAS4A, KRAS4B, NRAS, and HRAS belong to the protein family of GTPases, which are signaling proteins for cell proliferation, activated upon phosphorylation of their substrate GDP. It is thought that in order to signal, the protein dimerizes in the membrane.[47] Despite the fact that RAS is one of the most frequently mutated oncogenes in cancer, it has remained undruggable because of the picomolar affinity with which GDP binds to its active site. Allosteric inhibitors 
have been now developed, bringing new hope.[48] Although RAS binding to the membrane and the way it is allosterically modulated (by lipids, RAS oligomerization or other membrane-bound proteins) remains elusive, inhibiting RAS-membrane interactions could present a novel strategy for its allosteric modulation.[49]

Finally, $\alpha$-synuclein, an intrinsically disordered protein, adopts amyloid conformations that deposit within Lewis bodies in Parkinson's disease. Recent work has revealed that $\alpha$-synuclein-associated toxicity might be the consequence of abnormal interactions with membranes and regulation of synaptic vesicles.[50,51] In fact, oxidative modifications to $\alpha$-synuclein disrupt its binding to lipid membranes, which is critical for its function. While allostery is a well-established concept for membrane proteins as discussed above, it is not adequately described in the context of disordered proteins. However, it is proposed in the literature that allosteric regulation through modification of specific residues may regulate $\alpha$-synuclein function.[52]

\section{The role of lipids in allosteric modulation of transmembrane proteins}

Lipid recognition by transmembrane proteins goes beyond these protein classes and may be a general mechanism of membrane protein modulation. For example, Cong et al recently determined how lipid binding to the ammonia channel (AmtB) modulates its interaction with the regulatory protein GInK.[53] ${ }^{* *}$ Authors found that in the case of positive allosteric modulation, binding of lipids to AmtB shifts the Boltzmann distribution of the interconverting states of $A m t B$ by preferentially stabilizing an AmtB structure resembling with AmtB complexed with GlnK. On the other hand, when the lipid POPA is bound, the conformational ensemble shifts to stabilize the uncomplexed AmtB state, thus giving rise to negative allostery and not allowing binding to GlnK. Additionally, variable lipid tail lengths of phosphatidyl glycol and the stereochemistry of phosphatidylethanolamine tails were seen to alter the degree of allosteric modulation. In their follow-up study, they found that different lipid pairs display a variety of allosteric modulation for AmtB. In particular, the binding of phosphatidylethanolamine and cardiolipin-like molecules are allosteric modulators of AmtB and disruption of this binding abolishes the positive allosteric modulation. [54]

A recent study of the mitochondrial translocator protein TSPO reveals a dynamic monomer-dimer equilibrium of TSPO in the membrane mediated by cholesterol.[55] Binding of cholesterol to TSPO leads to structural changes across the protein that shifts the dynamic equilibrium towards the monomer protein. The study revealed an allosteric signal pathway that connects membrane protein tertiary and quaternary structure with cholesterol binding connecting the lower structural stability of the intervening transmembrane regions to provide a mechanistic basis for signal transmission. Recent advances in the study of phospholipases A2 (PLA2) enzymes show that three different major human PLA2s (CPLA2, IPLA2, sPLA2) achieve lipid head-group and acyl chain specificity by recruiting the optimal fatty acid at the sn-2 
position of a phospholipid molecule to their unique hydrophobic binding subsite.[56]**

An overview of the different mechanisms with which membrane proteins can be regulated allosterically can be seen in Figure 4.

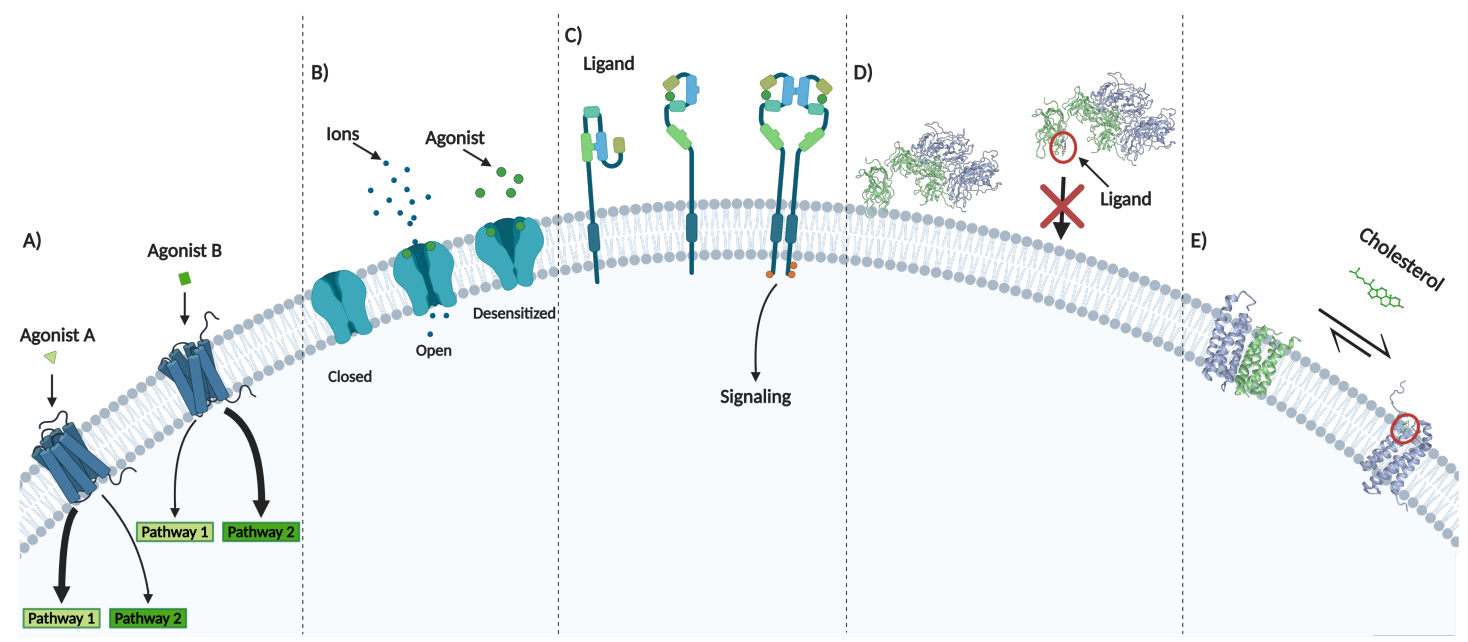

Figure 4. Overview of allosteric mechanisms in membrane proteins. A) Agonist A shifts the conformational equilibrium, stabilizing a state leading to Pathway 1 , while agonist $B$ stabilizes a different protein conformation resulting in a biased signal towards another pathway. B) The agonists (green circles) bind on the extracellular (EC) domain of a pLGIC, reducing the energy barrier of channel opening, blooming the EC domain. In high agonist concentrations the receptor although bound to its agonist has decreased response and is found in the desensitized state. C) On the left, the tethered monomeric apo EGFR dominates the conformational ensemble. Ligand binding induces large structural changes (allosteric phenomenon) on the EC domain, which favors homodimerization. D) A membrane-binding inhibitor of Coagulation Factor VIII discoidin domain (C2 domain) disrupts the proteinmembrane interaction (PDB: 3HNB).[57] E) The cholesterol depended monomer-dimer equilibrium of TSPO. Increase in cholesterol shifts the dynamic equilibrium towards the monomer state.

\section{Opportunities for Drug Design}

As an explosion of investigations of allosteric regulation of membrane proteins is materializing, the opportunity to modulate the protein-membrane interface should not be overlooked. The efficient targeting of protein-membrane interfaces can be considered as a novel drug design strategy as abnormal protein-membrane attachment is involved in deregulated cellular pathways and in disease. The feasibility of targeting the protein-membrane interface is supported by the fact that transmembrane proteins contain binding sites hosting lipids and peripheral membrane proteins have a membrane-binding domain, which could be potentially targeted by small molecules. Rational design of allosteric modulators holds big promise for drugging proteins that are considered undruggable but also for 
enhancing the activity (or the inhibition) of existing orthosteric drugs providing better selectivity and less side effects. $[49,58,59]$

\section{Conclusions}

Allostery is a biochemical mechanism of paramount importance for human life, having been termed as the "second secret of life," second only to the genome,[60] as it allows a multitude of flexibility for protein function, which can be regulated by sensing appropriate external signals. At the same time, membrane proteins are over $40 \%$ of the protein drug targets. Clearly, the combination of the two facts points to allostery potentially being a very promising avenue for creating novel modulators of membrane protein function. Reconsidering the accessibility of membrane-protein interfaces by drug-like molecules, which has been so far unexplored, may be a promising strategy for a new era in drugging membrane proteins.

\section{Acknowledgements}

Z.C. would like to acknowledge funding from the European Union's Horizon 2020 Framework Programme for Research and Innovation under Grant Agreement No. 785907 (Human Brain Project SGA2). A.C. is co-financed by Greece and the European Union (European Social Fund- ESF) through the Operational Programme «Human Resources Development, Education and Lifelong Learning» in the context of the project "Strengthening Human Resources Research Potential via Doctorate Research" (MIS-5000432), implemented by the State Scholarships Foundation (IKY).

1. Changeux JP, Christopoulos A: Allosteric modulation as a unifying mechanism for receptor function and regulation. Cell (2016) 166(5):1084-1102.

2. del Sol A, Tsai CJ, Ma B, Nussinov R: The origin of allosteric functional modulation: Multiple pre-existing pathways. Structure (2009) 17(8):1042-1050.

3. Bohr C, Hasselbalch $\mathrm{K}$, Krogh A: Ueber einen in biologischer beziehung wichtigen einfluss, den die kohlensäurespannung des blutes auf dessen sauerstoffbindung übt1. Skandinavisches Archiv Für Physiologie (1904) 16(2):402-412.

4. Hill AV: The possible effects of the aggregation of the molecules of hæmoglobin on its dissociation curves. J Physiol (1910) 40(i--vii.

5. Adair GS: The hemoglobin system. Iv. The reproduction of the carbon dioxide curves of blood with an artificial mixture of hemoglobin and sodium bicarbonate. J Biol Chem (1925) 63(2):515-516. 
6. Pauling L: The oxygen equilibrium of hemoglobin and its structural interpretation. $P$ Natl Acad Sci USA (1935) 21(4):186-191.

7. Klotz IM: The application of the law of mass action to binding by proteins; interactions with calcium. Arch Biochem (1946) 9(1):109117.

8. Monod J, Wyman J, Changeux JP: On the nature of allosteric transitions: A plausible model. J Mol Biol (1965) 12(88-118.

9. Koshland Jr D, Némethy G, Filmer D: Comparison of experimental binding data and theoretical models in proteins containing subunits. Biochemistry (1966) 5(1):365-385.

10. Cui Q, Karplus M: Allostery and cooperativity revisited. Protein Sci (2008) 17(8):1295-1307.

11. Motlagh HN, Wrabl JO, Li J, Hilser VJ: The ensemble nature of allostery. Nature (2014) 508(7496):331-339.

12. Tsai CJ, Nussinov R: A unified view of "how allostery works". PLoS computational biology (2014) 10(2):e1003394.

13. Gunasekaran $\mathrm{K}, \mathrm{Ma} \mathrm{B}$, Nussinov R: Is allostery an intrinsic property of all dynamic proteins? Proteins (2004) 57(3):433-443.

14. Hilser VJ, Wrabl JO, Motlagh HN: Structural and energetic basis of allostery. Annu Rev Biophys (2012) 41(585-609.

15. Kar G, Keskin O, Gursoy A, Nussinov R: Allostery and population shift in drug discovery. Curr Opin Pharmacol (2010) 10(6):715-722.

16. Böhm SK, Grady EF, Bunnett NW: Regulatory mechanisms that modulate signalling by g-protein-coupled receptors. The Biochemical journal (1997) 322 ( Pt 1)(Pt 1):1-18.

17. Mulle C, Benoit P, Pinset C, Roa M, Changeux JP: Calcitonin gene-related peptide enhances the rate of desensitization of the nicotinic acetylcholine receptor in cultured mouse muscle cells. Proc Natl Acad Sci U S A (1988) 85(15):5728-5732.

18. Schamel WW, Alarcon B, Hofer T, Minguet S: The allostery model of tcr regulation. J Immunol (2017) 198(1):47-52.

19. Bock A, Merten N, Schrage R, Dallanoce C, Batz J, Klockner J, Schmitz J, Matera C, Simon K, Kebig A, Peters L et al: The allosteric vestibule of a seven transmembrane helical receptor controls g-protein coupling. Nat Commun (2012) 3(1044. 
20. Lagerstrom MC, Schioth HB: Structural diversity of g protein-coupled receptors and significance for drug discovery. Nat Rev Drug Discov (2008) 7(4):339-357.

21. Kenakin T, Miller LJ: Seven transmembrane receptors as shapeshifting proteins: The impact of allosteric modulation and functional selectivity on new drug discovery. Pharmacol Rev (2010) 62(2):265304.

22. Bock A, Bermudez M, Krebs F, Matera C, Chirinda B, Sydow D, Dallanoce C, Holzgrabe U, De Amici M, Lohse MJ, Wolber G et al: Ligand binding ensembles determine graded agonist efficacies at a groteincoupled receptor. J Biol Chem (2016) 291(31):16375-16389.

23. Bermudez M, Nguyen TN, Omieczynski C, Wolber G: Strategies for the discovery of biased gpcr ligands. Drug Discovery Today (2019) 24(4):1031-1037.

24. Hilger D, Masureel M, Kobilka BK: Structure and dynamics of gpcr signaling complexes. Nat Struct Mol Biol (2018) 25(1):4-12.

25. Gurevich VV, Gurevich EV: Gpcr signaling regulation: The role of grks and arrestins. Frontiers in pharmacology (2019) 10 (125.

26. Kenakin T, Christopoulos A: Signalling bias in new drug discovery: Detection, quantification and therapeutic impact. Nat Rev Drug Discov (2013) 12(3):205-216.

27. Schrage R, Kostenis E: Functional selectivity and dualsteric/bitopic gpcr targeting. Curr Opin Pharmacol (2017) 32(85-90.

28. Szlenk CT, Gc JB, Natesan S: Does the lipid bilayer orchestrate access and binding of ligands to transmembrane orthosteric/allosteric sites of g protein-coupled receptors? Mol Pharmacol (2019) 96(5):527-541.

29. Ntountaniotis D, Kellici TF, Gkeka P, Cournia Z, Galdadas I, Mali G, BeckerBaldus J, Glaubitz C, Kriechbaum M, Rappolt M, Liapakis G et al: Drugmembrane interactions in the renin angiotensin system. In: Thermodynamics and biophysics of biomedical nanosystems. Demetzos C, Pippa N (Eds), Springer Nature, Singapore (2019):339364.

30. Guixa-Gonzalez R, Albasanz JL, Rodriguez-Espigares I, Pastor M, Sanz F, Marti-Solano M, Manna M, Martinez-Seara H, Hildebrand PW, Martin M, Selent J: Membrane cholesterol access into a g-protein-coupled receptor. Nat Commun (2017) 8(14505. *

Shows for the first time that cholesterol can occupy an orthosteric site in the A2A receptor using an evolutionarily conserved gateway. 
31. Oates J, Watts A: Uncovering the intimate relationship between lipids, cholesterol and gpcr activation. Curr Opin Struct Biol (2011) 21(6):802807.

32. Thal DM, Glukhova A, Sexton PM, Christopoulos A: Structural insights into g-protein-coupled receptor allostery. Nature (2018) 559(7712):45-53.

33. White JH, Wise A, Main MJ, Green A, Fraser NJ, Disney GH, Barnes AA, Emson $\mathrm{P}$, Foord SM, Marshall $\mathrm{FH}$ : Heterodimerization is required for the formation of a functional gabab receptor. Nature (1998) 396(679.

34. Borroto-Escuela DO, Fuxe K: Oligomeric receptor complexes and their allosteric receptor-receptor interactions in the plasma membrane represent a new biological principle for integration of signals in the cns. Front Mol Neurosci (2019) 12(230.**

A review summarizing the allosteric mechanisms governing the GPCR

oligomerization at the plasma membrane and their importance in CNS functions.

35. Taly A, Henin J, Changeux JP, Cecchini M: Allosteric regulation of pentameric ligand-gated ion channels: An emerging mechanistic perspective. Channels (Austin) (2014) 8(4):350-360.

36. Heusser SA, Lycksell M, Wang X, McComas SE, Howard RJ, Lindahl E:

Allosteric potentiation of a ligand-gated ion channel is mediated by access to a deep membrane-facing cavity. Proc Natl Acad Sci U S A (2018) 115(42):10672-10677.*

Latest research on the allosteric modulation of a pentameric ligand-gated ion channel shows that allostery is mediated by access to a deep membrane-facing cavity.

37. Prevost MS, Sauguet L, Nury H, Van Renterghem C, Huon C, Poitevin F, Baaden M, Delarue M, Corringer PJ: A locally closed conformation of a bacterial pentameric proton-gated ion channel. Nat Struct Mol Biol (2012) 19(6):642-649.

38. Sauguet L, Shahsavar A, Poitevin F, Huon C, Menny A, Nemecz A, Haouz A, Changeux JP, Corringer PJ, Delarue M: Crystal structures of a pentameric ligand-gated ion channel provide a mechanism for activation. Proc Natl Acad Sci U S A (2014) 111(3):966-971.

39. Fourati Z, Howard RJ, Heusser SA, Hu H, Ruza RR, Sauguet L, Lindahl E, Delarue M: Structural basis for a bimodal allosteric mechanism of general anesthetic modulation in pentameric ligand-gated ion channels. Cell Rep (2018) 23(4):993-1004.

40. Cerdan AH, Martin NE, Cecchini M: An ion-permeable state of the glycine receptor captured by molecular dynamics. Structure (2018) 26(11):1555-1562.e1554. 
41. Gielen M, Corringer PJ: The dual-gate model for pentameric ligandgated ion channels activation and desensitization. J Physiol (2018) 596(10):1873-1902.

42. Tsai CJ, Nussinov R: Emerging allosteric mechanism of egfr activation in physiological and pathological contexts. Biophys $J$ (2019) 117(1):513.

43. Leontiadou H, Galdadas I, Athanasiou C, Cournia Z: Insights into the mechanism of the pik3ca e545k activating mutation using MD simulations. Sci Rep (2018) 8(1):15544.

44. Zhang M, Jang $H$, Nussinov R: The mechanism of pi3k $\boldsymbol{\alpha}$ activation at the atomic level. Chemical Science (2019) 10(12):3671-3680.

45. Gkeka P, Evangelidis T, Pavlaki M, Lazani V, Christoforidis S, Agianian B, Cournia Z: Investigating the structure and dynamics of the pik3ca wild-type and H1047R oncogenic mutant. PLoS Comput Biol (2014) 10(10):e1003895.

46. Sethi A, Eargle J, Black AA, Luthey-Schulten Z: Dynamical networks in trna:Protein complexes. Proc Natl Acad Sci U S A (2009) 106(16):66206625.

47. Ambrogio C, Köhler J, Zhou Z-W, Wang H, Paranal R, Li J, Capelletti M, Caffarra C, Li S, Lv Q, Gondi S et al: Kras dimerization impacts mek inhibitor sensitivity and oncogenic activity of mutant kras. Cell (2018) 172(4):857-868.e815.

48. Kessler D, Gmachl M, Mantoulidis A, Martin LJ, Zoephel A, Mayer M, Gollner A, Covini D, Fischer S, Gerstberger T, Gmaschitz T et al: Drugging an undruggable pocket on kras. Proc Natl Acad Sci U S A (2019) 116(32):15823-15829.

49. Li Z, Buck M: Computational design of myristoylated cell-penetrating peptides targeting oncogenic k-ras.G12d at the effector-binding membrane interface. Journal of chemical information and modeling (2020) 60(1):306-315.

50. Auluck PK, Caraveo G, Lindquist S: A-synuclein: Membrane interactions and toxicity in parkinson's disease. Annual Review of Cell and Developmental Biology (2010) 26(1):211-233.

51. Iyer A, Claessens $\mathrm{M}$ : Disruptive membrane interactions of alphasynuclein aggregates. Biochim Biophys Acta Proteins Proteom (2019) 1867(5):468-482.

52. Sevcsik E, Trexler AJ, Dunn JM, Rhoades E: Allostery in a disordered protein: Oxidative modifications to alpha-synuclein act distally to 
regulate membrane binding. J Am Chem Soc (2011) 133(18):71527158.

53. Cong X, Liu Y, Liu W, Liang X, Laganowsky A: Allosteric modulation of protein-protein interactions by individual lipid binding events. Nat Commun (2017) 8(1):2203. **

Demonstrates how individual lipid-binding events can influence integral membrane protein and soluble protein interactions, using the AmtB-GlnK complex as a model system.

54. Patrick JW, Boone CD, Liu W, Conover GM, Liu Y, Cong X, Laganowsky A: Allostery revealed within lipid binding events to membrane proteins. Proc Natl Acad Sci U S A (2018) 115(12):2976-2981.

55. Jaipuria G, Leonov A, Giller K, Vasa SK, Jaremko L, Jaremko M, Linser R, Becker S, Zweckstetter M: Cholesterol-mediated allosteric regulation of the mitochondrial translocator protein structure. Nat Commun (2017) 8(14893.

56. Mouchlis VD, Chen Y, McCammon JA, Dennis EA: Membrane allostery and unique hydrophobic sites promote enzyme substrate specificity. J Am Chem Soc (2018) 140(9):3285-3291.**

Lipidomics coupled with molecular dynamics reveal unique phospholipase A2 specificity toward membrane phospholipid substrates - lipid headgroup as well as acylchain specificity for three major human phospholipases A2.

57. Liu Z, Lin L, Yuan C, Nicolaes GA, Chen L, Meehan EJ, Furie B, Furie B, Huang M: Trp2313-his2315 of factor viii c2 domain is involved in membrane binding: Structure of a complex between the $\mathrm{c} 2$ domain and an inhibitor of membrane binding. J Biol Chem (2010) 285(12):8824-8829.

58. Nussinov R, Tsai CJ: The different ways through which specificity works in orthosteric and allosteric drugs. Curr Pharm Design (2012) 18(9):1311-1316.

59. Segers K, Sperandio O, Sack M, Fischer R, Miteva MA, Rosing J, Nicolaes GA, Villoutreix BO: Design of protein membrane interaction inhibitors by virtual ligand screening, proof of concept with the $\mathbf{c} 2$ domain of factor v. Proc Natl Acad Sci U S A (2007) 104(31):12697-12702.

60. Fenton AW: Allostery: An illustrated definition for the 'second secret of life'. Trends Biochem Sci (2008) 33(9):420-425. 